ORIGINAL RESEARCH

\title{
Prevalence of Cancer Screening Among Adults With Disabilities, United States, 2013
}

\author{
C. Brooke Steele, $\mathrm{DO}^{1}$; Julie S. Townsend, $\mathrm{MS}^{1}$; Elizabeth A. Courtney-Long, MA, MSPH${ }^{2}$; \\ Monique Young, $\mathrm{MPH}^{1}$
}

\begin{abstract}
Suggested citation for this article: Steele CB, Townsend JS, Courtney-Long EA, Young M. Prevalence of Cancer Screening Among Adults With Disabilities, United States, 2013. Prev Chronic Dis 2017;14:160312. DOI: https://doi.org/10.5888/ pcd14.160312.
\end{abstract}

\section{PEER REVIEWED}

\section{Abstract}

\section{Introduction}

Many studies on cancer screening among adults with disabilities examined disability status only, which masks subgroup differences. We examined prevalence of receipt of cancer screening tests by disability status and type.

\section{Methods}

We used 2013 National Health Interview Survey data to assess prevalence of 1) guideline-concordant mammography, Papanicolaou (Pap) tests, and endoscopy and stool tests; 2) physicians' recommendations for these tests; and 3) barriers to health-care access among adults with and without disabilities (defined as difficulty with cognition, hearing, vision, or mobility).

\section{Results}

Reported Pap test use ranged from $66.1 \%$ (95\% confidence interval [CI], $60.3 \%-71.4 \%)$ to $80.2 \%$ (95\% CI, $72.4 \%-86.2 \%)$ among women with different types of disabilities compared with $81.4 \%$ (95\% CI, 80.0\%-82.7\%) among women without disabilities. Prevalence of mammography among women with disabilities was also lower (range, $61.2 \%$ [95\% CI, 50.5\%-71.0\%] to 67.5\% [95\% CI, 62.8\%-71.9\%]) compared with women without disabilities (72.8\% [95\% CI, 70.7\%-74.9\%]). Screening for colorectal cancer was $57.0 \%$ among persons without disabilities, and ranged from $48.6 \%$ (95\% CI, 40.3\%-57.0\%) among those with vision limita- tions to $64.6 \%(95 \%$ CI, $58.5 \%-70.2 \%)$ among those with hearing limitations. Receiving recommendations for Pap tests and mammography increased all respondents' likelihood of receiving these tests. The most frequently reported barrier to accessing health care reported by adults with disabilities was difficulty scheduling an appointment.

\section{Conclusion}

We observed disparities in receipt of cancer screening among adults with disabilities; however, disparities varied by disability type. Our findings may be used to refine interventions to close gaps in cancer screening among persons with disabilities.

\section{Introduction}

Cancer is the second leading cause of death among adults in the United States (1). Some cancers are preventable with regular screening tests and can be cured if detected and treated early. However, disparities in the use of preventive health services exist. People with disabilities have lower cancer screening rates than people without disabilities (2-7). In 2013, approximately 1 in 5 US adults reported having a disability; disabilities in mobility and cognition were the most frequently reported types (8). People with disabilities may have numerous health-care access barriers, including inaccessible health communication formats, limited access to transportation and parking, and lack of height-adjustable examination tables, accessible mammography equipment, and medical staff trained on proper patient lifting, transferring, and positioning techniques $(6,9-11)$.

People with disabilities are a heterogeneous group whose health needs vary with the types of limitations they have and by the nature of their disabilities (12). Definitions of disability and the level of functioning that qualifies for disability status vary. Many studies on cancer screening among this population used broad disability measures based on limitations in actions within the environment to compare prevalence of screening between people with 
and without disabilities, and a few disaggregated data by disability severity or type to look at subgroup differences (2-7,9-11,13-16).

In studies of up-to-date breast cancer screening, screening rates ranged from $67 \%$ to $79 \%$ among women with disabilities and from $70 \%$ to $83 \%$ among women without disabilities $(2,6,9,14)$. Receipt of up-to-date cervical cancer screening among women with disabilities ranged from $77 \%$ to $82 \%$, compared with a range of $83 \%$ to $87 \%$ among women without disabilities $(6,9,14)$. Breast and cervical cancer screening rates are lower among women with intellectual and developmental disabilities, cognitive disabilities, and multiple disabilities than among women with other disability types $(3,5,15)$. Numerous studies of colorectal cancer (CRC) screening among people with disabilities assessed whether a person was ever screened, rather than whether screening was guideline-concordant $(4,5,14,17)$. Some studies reported that CRC screening rates are higher among people with disabilities than among those without disabilities; however, rates varied by disability type $(14,17)$. Up-to-date CRC screening rates were recently published in a study examining receipt of tests from 1998 through 2010 among people with and without chronic disabilities; the authors reported that receipt was similar between both groups (18). The data in many earlier studies of cancer screening prevalence are now nearly a decade old or older and may not reflect recent trends. Additionally, few studies examined receipt of a health-care provider's recommendation for screening $(11,13,16)$.

In this study, we used a nationally representative sample to examine differences in receipt of guideline-concordant screening in 2013 for breast cancer, cervical cancer, and CRC by disability status and by type of disability. We report the prevalence of up-todate breast and cervical cancer screening among women with and without disabilities by screening recommendation status and the prevalence of receiving a recommendation for screening among adults with and without disabilities who were not up-to-date with CRC screening. In addition, we describe perceived barriers to health-care access by disability status.

\section{Methods}

We used data from the 2013 National Health Interview Survey (NHIS), a continuous, cross-sectional survey of US households conducted in-person with noninstitutionalized civilians. NHIS uses trained US Census Bureau interviewers and monitors for quality control. Sampling is done through a complex survey design that involves stratification, clustering, and multiple stages. One sample adult is selected in the household to provide additional information, which in 2013 included information for a cancer control supplement. The final adult response rate for the 2013 survey was
$61.2 \%$ (19). More information on NHIS design is available at http://www.cdc.gov/nchs/nhis.htm. This study did not require institutional review board approval because we used a publicly available data set without personal identifiers.

We included adults aged 21 to 75 years who provided responses to questions in the NHIS cancer control supplement and the disability module. The disability module consists of 6 questions, the following 4 of which measure serious functional limitations pertaining to hearing, vision, cognition, and mobility (20): Are you deaf or do you have serious difficulty hearing? Are you blind or do you have serious difficulty seeing even when wearing glasses? Because of a physical, mental, or emotional condition, do you have serious difficulty concentrating, remembering, or making a decision? Do you have serious difficulty walking or climbing stairs? In the 2013 survey, these questions were administered to approximately half of sample adults; household members serving as proxies for those who were unable to respond were excluded. Even though survey respondents could report more than one limitation, we excluded those with a mobility disability (eg, difficulty walking or climbing stairs) from the hearing, vision, and cognition categories. We did this because most people with multiple disabilities also have a mobility disability, and we wanted to make our estimates comparable with those in previous disability studies. The hearing, vision, and cognitive categories were not mutually exclusive, and respondents could be in more than one of these categories. Adults who answered no to all disability questions were considered not to have a serious functional limitation. We excluded from the analysis adults who reported having difficulty with self-care (eg, bathing, dressing) or having limitations with community involvement (eg, shopping or running errands alone) but did not report other disabilities $(n=65)$.

We merged the disability questions file, the sample adult file, and the person file to form the final analytic data set. The merged file had data on 15,079 adults aged 21 to 75 years with known disability status, 12,499 adults without a serious functional disability, and 2,580 adults with at least 1 of the 4 types of disability.

We included the following demographic variables: sex, race/ethnicity, marital status, education level, and general health status. Variables measuring whether respondents had health insurance coverage or usual sources of health care were used to assess health-care access. Barriers to care, such as difficulties with scheduling appointments and reaching clinic staff by telephone, long waiting-room times, inconvenient clinic hours, and lack of transportation served as proxies for barriers to cancer screening. The cancer control supplement included questions about receipt of screening tests for breast cancer, cervical cancer, and CRC. If a survey respondent indicated that he or she had received a screening test, then a follow-up question was asked about the timing of

\footnotetext{
The opinions expressed by authors contributing to this journal do not necessarily reflect the opinions of the U.S. Department of Health and Human Services, the Public Health Service, the Centers for Disease Control and Prevention, or the authors' affiliated institutions.
} 
the test. Additionally, women who had seen a clinician within the previous 12 months were asked whether a health-care professional recommended that they receive a mammogram or Papanicolaou (Pap) test. Adults who reported that they were not up to date with screening for CRC were asked whether a health-care provider had recommended that they be tested for problems in their colon or rectum within the previous 12 months. We used US Preventive Services Task Force recommendations that were in place in 2013 to define up-to-date cancer screening. For cervical cancer screening, we assessed whether women aged 21 to 65 years who did not report having had a hysterectomy had received a Pap test within the past 3 years. For breast cancer screening, we assessed whether women aged 50 to 74 years had received a mammogram within the past 2 years. Both men and women aged 50 to 75 years were considered appropriately screened for CRC and current with the screening recommendation if they had received either a fecal occult blood (FOBT) test within the previous year, sigmoidoscopy within the previous 5 years along with FOBT within the previous 3 years, or colonoscopy within the previous 10 years.

We used SAS 9.3 (SAS Institute Inc) and SAS-callable SUDAAN release 11 (Research Triangle Institute) to conduct all analyses. All prevalence estimates were weighted so that they represent the noninstitutionalized, civilian US population. The final survey weights account for race/ethnicity, sex, and age composition of that population.

We calculated the weighted prevalence estimates with $95 \%$ confidence intervals (CIs) for demographic characteristics, health-care access, and cancer screening (including by provider recommendation) among adults without a disability; separately for adults with hearing, vision, cognition, or mobility limitations; and for all adults with any of these disability types. We conducted multivariable logistic regression analysis to obtain adjusted odds ratios for the likelihood of being up-to-date for cancer screening for each type of disability (adults without disability served as the referent group for all models), while controlling for the influence of demographic characteristics and health-care access. In all models, we controlled for race/ethnicity, marital status, education level, health-care coverage, general health status (excluded in the hearing disability model for CRC screening because of statistical nonsignificance), and usual source of care. The cervical cancer screening model also controlled for age category, and the CRC screening model controlled for age category and sex. We used a backward elimination approach to select variables for the final models and assessed goodness of fit using the Hosmer-Lemeshow test. Variables with $P \geq .10$ from the Wald $F$ test were eliminated unless they traditionally appeared in cancer screening models.

\section{Results}

Overall, $16.9 \%$ of adults aged 21 to 75 years were identified as having at least 1 of the 4 disability types. The prevalence of each disability type among this sample was mobility, $57.9 \%$; cognition, $18.2 \%$; hearing, $18.8 \%$; and vision, $11.2 \%$. Thirty-two percent of persons had more than one disability, with most $(82.8 \%)$ having mobility limitations in combination with another disability. Regardless of disability type or status, most adults were non-Hispanic white and had health insurance coverage and a usual source of care (Table 1). Compared with persons with no disability $(6.4 \%)$, the prevalence of reporting fair or poor health was substantially higher among persons with a mobility disability $(63.3 \%)$, a cognitive disability (38.4\%), a vision disability $(29.4 \%)$, or a hearing disability (17.4\%).

In unadjusted prevalence estimates (Table 2), women with any of the disability types had a lower prevalence of reporting up-to-date cervical and breast cancer screening than women without a disability. However, men and women with any of the disability types had a slightly higher prevalence of up-to-date CRC screening than adults without disabilities. Women with mobility limitations had the lowest prevalence $(66.1 \%)$ of receiving a Pap test within the past 3 years, whereas those with cognitive limitations had the highest $(80.2 \%)$. The reverse was true for receipt of mammograms within the past 2 years; prevalence was lower among women with cognitive limitations $(61.2 \%)$ and higher among those with mobility limitations $(67.5 \%)$. When controlling for sociodemographic and health-care-related variables we found that compared with women without disability, the odds of receiving a Pap test within the previous 3 years were significantly lower among women with any of the disability types (AOR, 0.77; 95\% CI, 0.60-0.99) and among women with a mobility limitation (AOR, 0.58; 95\% CI, 0.42-0.80). The odds of receiving mammograms within the previous 2 years were also lower among women with any of the disability types; however, this finding was not significant.

The prevalence of up-to-date breast and cervical cancer screening was higher among women who reported receiving recommendations from their health-care providers for these tests than among women who said they had not received recommendations (Figure). Among women who reported receiving recommendations, those with any of the disability types were less likely than those without any of the disability types to report receiving Pap tests within the previous 3 years $(84.1 \%$ vs $94.7 \%$, respectively, $P<.001)$. Among women who indicated that they had not received recommendations, those with any of the disability types reported less use of mammography $(32.6 \%$ vs $48.6 \%, P<.001)$ and of Pap tests $(55.2 \%$ vs $66.7 \%, P=.003)$ than women without any of the disab-

\footnotetext{
The opinions expressed by authors contributing to this journal do not necessarily reflect the opinions of the U.S. Department of Health and Human Services, the Public Health Service, the Centers for Disease Control and Prevention, or the authors' affiliated institutions.
} 
ility types. Among adults who reported that they were not up-todate with screening for $\mathrm{CRC}$, the percentage who had received recommendations for CRC screening tests in the previous 12 months was $15.2 \%$ for those with any of the disability types and $11.9 \%$ for those without any of the disability types $(P>.05)$ (data not shown). The prevalence of perceived barriers to accessing health care was higher among persons with any of the disability types than persons without any of the disability types; the most frequently cited barrier among both groups was difficulty getting a clinic appointment $(9.1 \%$ vs $4.5 \%)$ (Table 3$)$.

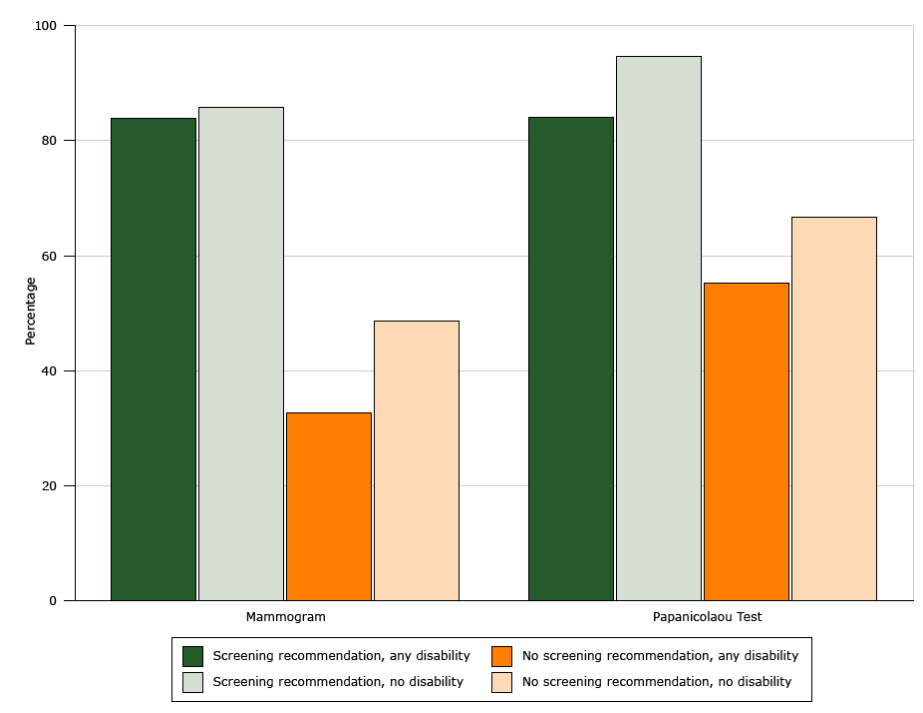

Figure. Prevalence of up-to-date cancer screening among women, by disability status and whether or not a doctor or health professional recommended the screening test, National Health Interview Survey, United States, 2013.

\section{Discussion}

Overall, our findings on cancer screening among persons with disabilities are consistent with some previous research on this topic $(2,3,14,16)$. We found a lower prevalence of breast and cervical cancer screening among women with disabilities, but only the disparity in Pap test use persisted in models adjusted for cancer screening-related variables. A similar pattern was reported in a study on use of mammography and Pap tests by disability status and severity (3). In a study examining receipt of these tests among women with and without limitations in activities of daily living, however, disparities remained significant in multivariable analyses for each test (14). Similar to previous research on CRC screening, we found slightly higher rates of screening among adults with disabilities than among those without disabilities $(14,18)$. Even though persons with disabilities were reported to have higher CRC screening rates than persons without disabilities from 1998 to 2010, the rates reported in 2010 (59.2\% vs 58.9\%) and in our study (61.8\% vs 57\%) are below the $70.5 \%$ Healthy People 2020 objective (https:/www.healthypeople.gov/2020/topics-objectives/topic/cancer/objectives) (18). The breast and cervical cancer screening rates in our study are also lower than national objectives for these tests $(81.1 \%$ for breast cancer, $93.0 \%$ for cervical cancer). Future studies should explore the reasons why some persons with disabilities may avoid or delay cancer screening, including the extent to which factors such as disability type, severity of limitation, and confusion about screening guidelines affect decision making. Research on this topic is scarce, especially for cervical cancer and CRC.

We found that the prevalence of breast and cervical cancer screening was higher among women who reported receiving recommendations for these tests from their health-care providers, which is consistent with previous studies $(11,13)$. Women with disabilities were slightly less likely than those without disabilities to report receipt of screening recommendations, but the differences were not significant. Less than $15 \%$ of persons who were not upto-date with CRC screening reported receiving recommendations for these tests, regardless of disability status. We did not find other studies that examined receipt of recommendations for CRC screening among this population. Additional research is needed to help identify the reasons why health-care providers might not refer persons with disabilities for cancer screening. Some providers may prioritize managing the disabling condition and related illnesses to the exclusion of addressing preventive health needs $(10,14)$. Tools have been developed to help clinicians identify recommended preventive services and increase their use among persons with disabilities (21-23). Information is also available for health insurers, community-based organizations, and health educators to help increase their knowledge about disability and health and identify service gaps (21).

We found that most people with disabilities had health insurance coverage and a usual source of health care. This finding is consistent with previous research and may be related to links between enrollment in federal and state disability benefits programs and eligibility for public health insurance $(2,3,8,24-26)$. However, some insured people with disabilities reported unmet health-care needs and difficulty accessing care. These disparities may be related to disability type (eg, mobility limitations), or they may be unrelated to disability status (eg, out-of-pocket cost) $(24,27)$. In our study, people with disabilities were 7 times as likely to report transportation barriers as those without disabilities and nearly twice as likely to report difficulty scheduling appointments, long wait times at clinics, and inconvenient clinic hours.

Our study has limitations. NHIS data are based on self-report and may be subject to recall bias, particularly among persons with serious cognitive disabilities, and subject to social desirability bias.

\footnotetext{
The opinions expressed by authors contributing to this journal do not necessarily reflect the opinions of the U.S. Department of Health and Human Services, the Public Health Service, the Centers for Disease Control and Prevention, or the authors' affiliated institutions.
} 
The questions used to measure disability in the NHIS disability module, however, have been cognitively tested by the National Center for Health Statistics and the US Census Bureau (28). Additionally, self-reported data for mammography, Pap tests, and CRC screening are reported to be reliable $(29,30)$. The small sample sizes for some disability subgroups limit the generalizability of our findings, but the sizes all met criteria for analysis; the data are also weighted to be representative of the US population. Nearly onethird of respondents had more than one type of disability; therefore, our results might not be generalizable to those with a single disability. Because we could not distinguish between tests received for diagnostic purposes and tests received for screening purposes, actual cancer screening rates may be lower than reported. We also could not assess the onset of disability in relation to receipt of screening tests. Using data based on disability status at the time of data collection rather than the time of screening could have resulted in overestimates of screening among those whose disabilities were diagnosed after they were screened. Furthermore, because the NHIS disability survey includes only noninstitutionalized adults living in the community, we could not assess the prevalence of cancer screening among adults living in group homes and other settings in which the prevalence of disability may be higher.

One of the strengths of our study is that we used population-based data. We also examined 2 cancer screening-related topics that have been rarely researched among persons with disabilities - reported receipt of guideline-concordant CRC screening and recommendations by health-care providers for cancer screening.

Disparities among persons with disabilities in receipt of preventive services and health-care access persist. Our study findings may be used to increase awareness about gaps in cancer screening among subgroups of this population, to inform development of interventions that educate people with disabilities about the importance of discussing preventive health services with their health care providers, and to remind providers about the critical roles they play in recommending use of these services.

\section{Acknowledgments}

This research received no specific grant from any funding agency in the public, private, or nonprofit sectors. The findings and conclusions in this report are those of the authors and do not necessarily represent the official position of the Centers for Disease Control and Prevention.

\section{Author Information}

Corresponding Author: C. Brooke Steele, DO, Centers for Disease Control and Prevention, 4770 Buford Hwy NE, MS F76, Atlanta, GA 30341.Telephone: 770-488-4261. E-mail: BSteele1@cdc.gov.

Author Affiliations: ${ }^{1}$ Division of Cancer Prevention and Control, Centers for Disease Control and Prevention, Atlanta, Georgia. ${ }^{2}$ Division of Human Development and Disability, Centers for Disease Control and Prevention, Atlanta, Georgia.

\section{References}

1. US Cancer Statistics Working Group. United States cancer statistics: 1999-2011 incidence and mortality web-based report. Atlanta (GA): US Department of Health and Human Services, Centers for Disease Control and Prevention, and National Cancer Institute; 2014. http://www.cdc.gov/uscs. Accessed May 11, 2015.

2. Courtney-Long E, Armour B, Frammartino B, Miller J. Factors associated with self-reported mammography use for women with and women without a disability. J Womens Health (Larchmt) 2011;20(9):1279-86.

3. Horner-Johnson W, Dobbertin K, Andresen EM, Iezzoni LI. Breast and cervical cancer screening disparities associated with disability severity. Womens Health Issues 2014; 24(1):e147-53.

4. Horner-Johnson W, Dobbertin K, Lee JC, Andresen EM. Rural disparities in receipt of colorectal cancer screening among adults ages 50-64 with disabilities. Disabil Health J 2014; 7(4):394-401.

5. Horner-Johnson W, Dobbertin K, Lee JC, Andresen EM; Expert Panel on Disability and Health Disparities. Disparities in health care access and receipt of preventive services by disability type: analysis of the Medical Expenditure Panel Survey. Health Serv Res 2014;49(6):1980-99.

6. Horner-Johnson W, Dobbertin K, Iezzoni LI. Disparities in receipt of breast and cervical cancer screening for rural women age 18 to 64 with disabilities. Womens Health Issues 2015; 25(3):246-53.

7. Abrams MT, Myers CS, Feldman SM, Boddie-Willis C, Park J, McMahon RP, et al. Cervical cancer screening and acute care visits among Medicaid enrollees with mental and substance use disorders. Psychiatr Serv 2012;63(8):815-22.

8. Courtney-Long EA, Carroll DD, Zhang QC, Stevens AC, Griffin-Blake S, Armour BS, et al. Prevalence of disability and disability type among adults — United States, 2013. MMWR Morb Mortal Wkly Rep 2015;64(29):777-83.

\footnotetext{
The opinions expressed by authors contributing to this journal do not necessarily reflect the opinions of the U.S. Department of Health and Human Services, the Public Health Service, the Centers for Disease Control and Prevention, or the authors' affiliated institutions.
} 
9. Armour BS, Thierry JM, Wolf LA. State-level differences in breast and cervical cancer screening by disability status: United States, 2008. Womens Health Issues 2009; 19(6):406-14.

10. Merten JW, Pomeranz JL, King JL, Moorhouse M, Wynn RD. Barriers to cancer screening for people with disabilities: a literature review. Disabil Health J 2015;8(1):9-16.

11. Yankaskas BC, Dickens P, Bowling JM, Jarman MP, Luken K, Salisbury $\mathrm{K}$, et al. Barriers to adherence to screening mammography among women with disabilities. Am J Public Health 2010;100(5):947-53.

12. Krahn GL, Walker DK, Correa-De-Araujo R. Persons with disabilities as an unrecognized health disparity population. Am J Public Health 2015;105(52,Suppl 2):S198-206.

13. Ramirez A, Farmer GC, Grant D, Papachristou T. Disability and preventive cancer screening: results from the 2001 California Health Interview Survey. Am J Public Health 2005; 95(11):2057-64.

14. Wei W, Findley PA, Sambamoorthi U. Disability and receipt of clinical preventive services among women. Womens Health Issues 2006;16(6):286-96.

15. Parish SL, Swaine JG, Son E, Luken K. Determinants of cervical cancer screening among women with intellectual disabilities: evidence from medical records. Public Health Rep 2013;128(6):519-26.

16. Drew JA, Short SE. Disability and Pap smear receipt among US women, 2000 and 2005. Perspect Sex Reprod Health 2010; 42(4):258-66.

17. Miller NA, Kirk A, Alston B, Glos L. Effects of gender, disability, and age in the receipt of preventive services. Gerontologist 2014;54(3):473-87.

18. Iezzoni LI, Kurtz SG, Rao SR. Trends in colorectal cancer screening over time for persons with and without chronic disability. Disabil Health J 2016;9(3):498-509.

19. National Center for Health Statistics. 2013 National Health Interview Survey public use data release: National Health Interview Survey description. Hyattsville (MD): US Department of Health and Human Services, Centers for Disease Control and Prevention, National Center for Health Statistics; 2014. ftp://ftp.cdc.gov/pub/Health_Statistics/NCHS/ Dataset_Documentation/NHIS/2013/srvydesc.pdf. Accessed March 1, 2016.

20. Altman B, Bernstein A. Disability and health in the United States, 2001-2005. Hyattsville (MD): National Center for Health Statistics; 2008.

21. Sinclair LB, Taft KE, Sloan ML, Stevens AC, Krahn GL. Tools for improving clinical preventive services receipt among women with disabilities of childbearing ages and beyond. Matern Child Health J 2015;19(6):1189-201.
22. American College of Obstetricians and Gynecologists Interactive Site for Clinicians Serving Women with Disabilities. http://www.acog.org/About-ACOG/ACOGDepartments/Women-with-Disabilities/Interactive-site-forclinicians-serving-women-with-disabilities. Accessed June 19, 2015.

23. Association for Maternal and Child Health Programs. Toolbox: improving the receipt of clinical and preventive services among women with disabilities. http://www.amchp.org/ programsandtopics/womens-health/Focus\%20Areas/ WomensHealthDisability/Pages/default.aspx. Accessed April $5,2016$.

24. Iezzoni LI, Frakt AB, Pizer SD. Uninsured persons with disability confront substantial barriers to health care services. Disabil Health J 2011;4(4):238-44.

25. Smith JC, Medalia CUS. Census Bureau, Current population reports, P60-253, Health insurance coverage in the United States: 2014. Washington (DC): US Government Printing Office; 2015.

26. Dobbertin K, Horner-Johnson W, Lee JC, Andresen EM. Subgroup differences in having a usual source of health care among working-age adults with and without disabilities. Disabil Health J 2015;8(2):296-302.

27. Henning-Smith C, McAlpine D, Shippee T, Priebe M. Delayed and unmet need for medical care among publicly insured adults with disabilities. Med Care 2013;51(11):1015-9.

28. Miller K, DeMaio TJ. Report on cognitive research of proposed American Community Survey disability questions. http://census.gov/srd/papers/pdf/ssm2006-06.pdf. Accessed May 11, 2015.

29. Baier M, Calonge N, Cutter G, McClatchey M, Schoentgen S, Hines S, et al. Validity of self-reported colorectal cancer screening behavior. Cancer Epidemiol Biomarkers Prev 2000; 9(2):229-32.

30. Caplan LS, McQueen DV, Qualters JR, Leff M, Garrett C, Calonge N. Validity of women's self-reports of cancer screening test utilization in a managed care population. Cancer Epidemiol Biomarkers Prev 2003;12(11 Pt 1):1182-7.

\footnotetext{
The opinions expressed by authors contributing to this journal do not necessarily reflect the opinions of the U.S. Department of Health and Human Services, the Public Health Service, the Centers for Disease Control and Prevention, or the authors' affiliated institutions.
} 


\section{Tables}

Table 1. Prevalence of Selected Demographic Characteristics and Health Behaviors Among Adults Aged 21-75 Years ( $N=15,079)$, by Disability Type ${ }^{a}$, National Health Interview Survey, United States, 2013

\begin{tabular}{|c|c|c|c|c|c|c|}
\hline \multirow[b]{2}{*}{ Characteristic } & \multicolumn{6}{|c|}{ Type of Disability, \% (95\% Confidence Interval) } \\
\hline & Hearing & Vision & Cognitive & Mobility & Any Type & None \\
\hline Total & 454 & 304 & 471 & 1,512 & 2,580 & 12,49 \\
\hline
\end{tabular}

Sex

Male

Female

Age group, $y$

21-49

50-64

65-75

Race/ethnicity

Non-Hispanic white

Non-Hispanic black

Hispanic

Other $^{\mathrm{b}}$

$62.5(57.2-67.6) \quad 47.9(41.5-54.3)$

$37.5(32.4-42.8) \quad 52.1(45.7-58.5)$

$47.8(42.2-53.4)$

$43.4(40.4-46.4)$

47.5 (45.3-49.7)

$56.6(53.6-59.6)$

$52.5(50.3-54.7)$

$46.8(45.7-47.9)$

$53.2(52.1-54.3)$

\begin{tabular}{|l|l|l|l|l|l|}
\hline $27.3(23.0-32.1)$ & $42.7(36.2-49.5)$ & $53.6(48.2-59.0)$ & $21.3(18.9-24.0)$ & $29.7(27.6-31.9)$ & $60.9(59.5-61.7)$ \\
\hline
\end{tabular}

\begin{tabular}{|c|r|r|r|r|r|}
\hline $35.7(31.1-40.6)$ & $36.1(30.2-42.4)$ & $33.0(28.0-38.4)$ & $46.5(43.7-49.4)$ & $41.4(39.3-43.7)$ & $27.0(26.0-27.9)$ \\
\hline
\end{tabular}

\begin{tabular}{|l|l|l|l|l|l|}
\hline $37.0(32.2-42.0)$ & $21.2(16.2-27.3)$ & $13.4(10.4-17.0)$ & $32.2(29.4-35.1)$ & $28.9(26.9-30.9)$ & $12.4(11.7-13.2)$ \\
\hline
\end{tabular}

Marital status

Married/living together

$81.2(77.3-84.5) \quad 60.5(53.8-66.7)$

Single/never married

Divorced/widowed/ separated

$5.8(4.1-8.2) \quad 14.7(10.8-19.6)$

\begin{tabular}{ll|}
$7.7(5.6-10.6)$ & $18.6(14.4-23.7)$
\end{tabular}

$5.3(3.6-7.7)$

$8.6(14.4-23.7)$
$-{ }^{\mathrm{c}}$

$70.6(65.9-74.9)$

$14.8(12.0-18.1)$

$9.9(7.4-13.2)$

$4.8(3.2-7.1)$

$66.1(63.1-68.9)$

69.4

$4(67.2-71.5)$

$66.7(65.6-67.7)$

\begin{tabular}{l|l}
$15.4(16.1-21.0)$ & $15.1(13.5-16.9)$ \\
\hline
\end{tabular}

$11.9(11.2-12.6)$

$10.7(9.1-12.5) \quad 10.6(9.3-12.1)$

$4.9(3.8-6.2)$

$4.9(4.0-5.9)$

$14.0(13.3-14.7)$

$7.4(6.9-8.0)$

\section{Education}

Less than high school diploma

High school diploma/GED

Some college/ associates degree

Bachelor's degree or higher

\begin{tabular}{|l|l|l|l|l|l|}
\hline $51.0(45.4-56.5)$ & $37.9(32.1-44.0)$ & $29.0(24.3-34.3)$ & $40.1(37.0-43.2)$ & $40.5(38.2-42.9)$ & $54.8(53.6-56.0)$ \\
\hline $17.5(13.6-22.2)$ & $22.1(16.9-28.4)$ & $35.9(30.7-41.4)$ & $16.4(14.3-18.7)$ & $19.9(18.0-21.9)$ & $24.6(23.5-25.8)$ \\
\hline $31.5(26.5-37.0)$ & $40.0(34.1-46.2)$ & $35.1(30.2-40.2)$ & $43.5(40.6-46.5)$ & $39.6(37.4-41.8)$ & $20.5(19.7-21.4)$ \\
\hline
\end{tabular}

General health status

Excellent/very good

Good

Fair/poor

$$
18.4(14.5-23.0)(22.7(18.0-28.3)
$$

\begin{tabular}{|r|r|r|r|r|r|}
\hline $18.4(14.5-23.0)$ & $22.7(18.0-28.3)$ & $22.4(18.9-26.4)$ & $25.1(22.4-28.0)$ & $23.2(21.4-25.1)$ & $9.9(9.3-10.5)$ \\
\hline $24.9(20.6-29.8)$ & $32.5(27.0-38.5)$ & $31.3(27.0-36.0)$ & $31.0(28.0-34.3)$ & $30.0(27.8-32.3)$ & $24.1(23.1-25.1)$ \\
\hline $30.9(26.1-36.2)$ & $26.3(21.2-32.1)$ & $29.5(24.9-34.5)$ & $31.1(28.2-34.1)$ & $30.1(28.1-32.2)$ & $31.2(30.2-32.3)$ \\
\hline $25.8(21.1-31.3)$ & $18.5(13.6-24.6)$ & $16.8(12.9-21.5)$ & $12.8(10.8-15.1)$ & $16.7(15.0-18.5)$ & $34.8(33.6-36.0)$ \\
\hline
\end{tabular}

$16.8(12.9-21.5)$

\section{Has insurance coverage}

Yes

\begin{tabular}{|r|r|r|r|r|r|}
\hline $47.5(42.0-53.2)$ & $37.3(30.6-44.5)$ & $23.5(19.1-28.7)$ & $11.7(9.8-13.8)$ & $22.8(20.7-25.0)$ & $68.6(67.6-69.5)$ \\
\hline $35.1(30.2-40.4)$ & $33.4(27.8-39.5)$ & $38.1(33.3-43.0)$ & $25.1(22.6-27.7)$ & $29.4(27.5-31.5)$ & $25.1(24.2-26.0)$ \\
\hline $17.4(13.4-22.2)$ & $29.4(23.9-35.5)$ & $38.4(33.3-43.8)$ & $63.3(60.4-66.1)$ & $47.8(45.3-50.2)$ & $6.4(5.9-6.9)$ \\
\hline
\end{tabular}

\begin{tabular}{|l|l|l|l|l|l|}
\hline $86.5(82.7-89.6)$ & $80.7(75.7-84.9)$ & $81.9(77.1-85.9)$ & $89.4(87.4-91.1)$ & $87.0(85.4-88.4)$ & $82.3(81.5-83.1)$ \\
\hline
\end{tabular}

Has usual source of care

Yes

$85.9(81.8-89.2) \quad 79.5(72.6-85.1)$

$88.3(84.4-91.3)$

$93.8(92.2-95.1)$

$90.3(88.8-91.6)$

$82.8(81.9-83.6)$

Abbreviation: GED, general educational development.

${ }^{a}$ Respondents could report more than one limitation and were included in the analysis for each reported limitation, with the exception of mobility limitations. Regardless of any additional limitation, people with mobility limitations were only included in the mobility limitation subgroup.

${ }^{b}$ American Indian/Alaska Native, Asian, multiple races, and race group not releasable.

${ }^{\mathrm{c}}$ Estimate suppressed because relative standard error was greater than $30 \%$.

The opinions expressed by authors contributing to this journal do not necessarily reflect the opinions of the U.S. Department of Health and Human Services, the Public Health Service, the Centers for Disease Control and Prevention, or the authors' affiliated institutions. 
Table 2. Prevalence and Adjusted Odds Ratios ${ }^{a}$ for Up-to-Date Cancer Screening Among Adults With a Disability Compared With Adults With No Disability, National Health Interview Survey, United States, 2013

\begin{tabular}{|c|c|c|c|c|c|c|}
\hline \multirow[b]{2}{*}{ Characteristic } & \multicolumn{6}{|c|}{ Disability Type $^{b}$} \\
\hline & Hearing & Vision & Cognitive & Mobility & Any Type & None \\
\hline \multicolumn{7}{|l|}{ Pap test } \\
\hline Pap test within past 3 years $^{\mathrm{c}}, \mathrm{n}$ & 90 & 94 & 166 & 392 & 700 & 5,184 \\
\hline Yes & $73.1(61.9-81.9)$ & $76.6(64.8-85.3)$ & $80.2(72.4-86.2)$ & $66.1(60.3-71.4)$ & $71.5(67.4-75.2)$ & $81.4(80.0-82.7)$ \\
\hline AOR for up-to-date status & $0.82(0.44-1.53)$ & $0.99(0.51-1.90)$ & $1.20(0.71-2.03)$ & $0.58(0.42-0.80)$ & $0.77(0.60-0.99)$ & Reference \\
\hline \multicolumn{7}{|l|}{ Mammogram } \\
\hline Mammogram within past 2 years ${ }^{\mathrm{d}}, \mathrm{n}$ & 115 & 80 & 107 & 633 & 897 & 2,544 \\
\hline Yes & $66.5(55.4-76.1)$ & $63.7(50.4-75.2)$ & $61.2(50.5-71.0)$ & $67.5(62.8-71.9)$ & $66.7(63.0-70.2)$ & $72.8(70.7-74.9)$ \\
\hline AOR for up-to-date status & $0.89(0.53-1.49)$ & $0.88(0.48-1.61)$ & $0.91(0.54-1.55)$ & $1.04(0.77-1.40)$ & $0.97(0.76-1.25)$ & Reference \\
\hline \multicolumn{7}{|l|}{ Colorectal cancer screening } \\
\hline Colorectal cancer screening ${ }^{\mathrm{e}}, \mathrm{n}$ & 320 & 175 & 216 & 1,136 & 1,746 & 4,726 \\
\hline Yes & $64.6(58.5-70.2)$ & $48.6(40.3-57.0)$ & $56.2(47.9-64.2)$ & $63.1(60.0-66.1)$ & $61.8(59.1-64.5)$ & $57.0(55.3-58.6)$ \\
\hline Received colonoscopy ${ }^{f}, \mathrm{n}$ & $93.1(87.5-96.3)$ & $92.8(84.5-96.8)$ & $93.6(86.3-97.2)$ & $93.5(91.2-95.3)$ & $93.5(91.5-95.0)$ & $94.7(93.7-95.6)$ \\
\hline AOR for up-to-date status & $1.41(1.04-1.91)$ & $0.90(0.60-1.35)$ & $1.25(0.84-1.85)$ & $1.33(1.12-1.58)$ & $1.29(1.10-1.52)$ & Reference \\
\hline
\end{tabular}

Abbreviation: AOR, adjusted odds ratio; Pap, Papanicolaou.

${ }^{\text {a }}$ Adjusted odds ratios are from logistic regression analyses that examined disability status in relation to being up-to-date on cancer screening tests while controlling for race/ethnicity, insurance, having a usual source of health care, general health status, marital status, and education. Colorectal cancer screening models also included sex and age category. General health status was not included in the hearing disability model because it was not significant. Pap test models also were controlled for age category.

${ }^{\mathrm{b}}$ Values are percentage (95\% confidence interval) unless otherwise stated.

${ }^{\mathrm{c}}$ Women aged 21 to 65 years who had a Pap test within the past 3 years. Data on Pap tests were available for 5,884 women.

${ }^{d}$ Women aged 50 to 74 years who had a mammogram within the past 2 years. Data on mammograms were available for 3,441 women.

${ }^{\mathrm{e}}$ Adults aged 50 to 75 years who had a high-sensitivity fecal occult blood test within the past 12 months, a sigmoidoscopy within the past 5 years with a highsensitivity fecal occult blood test within the past 3 years, or a screening colonoscopy within the past 10 years. Data on colorectal cancer screening were available for 6,472 men and women.

${ }^{f}$ Colonoscopy within the past 10 years among adults with known disability status who reported being up-to-date with colorectal cancer screening $(n=3,677)$.

The opinions expressed by authors contributing to this journal do not necessarily reflect the opinions of the U.S. Department of Health and Human Services, the Public Health Service, the Centers for Disease Control and Prevention, or the authors' affiliated institutions. 
Table 3. Perceived Barriers to Health Care Access by Disability Status $\left(N=12,499^{\mathrm{a}}\right)$, National Health Interview Survey, United States, 2013

\begin{tabular}{|l|r|r|}
\hline \multirow{2}{*}{ Barrier } & \multicolumn{2}{|c|}{ Any Disability } \\
\cline { 2 - 3 } D & \multicolumn{2}{|c|}{ \% (95\% Confidence Interval) } \\
\hline Difficulty getting through on telephone to reach clinic & $4.2(3.4-5.2)$ & $1.6(1.4-1.9)$ \\
\hline Difficulty getting clinic appointment & $9.1(7.9-10.6)$ & $7.3(6.1-8.7)$ \\
\hline Wait time at clinic too long & $4.5(4.1-4.9)$ \\
\hline Clinic not open at convenient times & $2.8(2.5-3.2)$ \\
\hline No available transportation to clinic & $2.3(3.5-5.4)$ & $6.5(5.4-7.8)$ \\
\hline
\end{tabular}

${ }^{a}$ The denominator for each survey question varies because of the exclusion of persons with unknown and missing responses.

${ }^{\mathrm{b}}$ Includes hearing, vision, cognitive, or mobility disability. 